\title{
Characterizing Optimism and Pessimism Directly through Comonotonicity
}

\author{
PETER WAKKeR* \\ Fuqua School of Business, Duke University, \\ Durham, North Carolina 27706
}

Received March 31, 1989; revised January 2, 1990

\begin{abstract}
Pessimism-independence is introduced to characterize pessimistic risk attitudes for the nonlinear-probability models of Schmeidler, Quiggin, and Yaari directly in terms of comonotonicity, rather than through additional conditions such as convexity of preferences. Pessimism-independence requires the mixture of an arbitrary good and a fixed act to be preferred to the mixture of a comonotonic bad act and the fixed act. Thus, more general than full-force independence, it does not exclude the additional (pessimistic) appreciation of the hedging involved in the mixture of a noncomonotonic bad and fixed act. More restrictive than comonotonic independence, it does exclude (optimistic) aversion of hedging. Journal of Economic Literature Classification Numbers: 026, 213,521. (C) 1990 Academic Press, Inc.
\end{abstract}

\section{INTRODUCTION}

This note gives a very simple, but (it is hoped) elegant, way to characterize optimism and pessimism in the nonexpected utility models of Schmeidler [15], Quiggin [13], and Yaari [24]. These models deal with nonlinear probabilities. The main new phenomenon that these approaches can incorporate is a kind of pessimism, excluded by traditional (additive) expected utility. In the nonlinear-probability models pessimism, and its counterpart optimism, have so far been characterized through additional conditions like convexity/concavity of preference relations, or aversion/ preference for mean-preserving increases in risk (for the latter see [4]). We show that a natural characterization can be obtained directly in terms of comonotonicity, a condition needed anyhow in the characterizations of nonlinear-probability models. Table I at the end of Section 4 summarizes the results of this paper.

* The research has been made possible by a fellowship of the Royal Netherlands Academy of Arts and Sciences, and a fellowship of the Netherlands Organization for Scientific Research. 
Section 2 gives some mathematical properties of the Choquet integral that will make possible our characterization of optimism and pessimism. Section 3 describes Schmeidler's [15] set-up, his new idea of comonotonic independence, and his main theorem. Section 4 starts with an intuitive interpretation of comonotonicity. We hope our way of characterizing pessimism/optimism will then appear natural to the reader. Adaptation to Yaari's set-up will also be indicated. The Appendix gives mathematical generalizations and elaborations, not given in the main body of the note for reasons of accessibility.

\section{CONCAVITy/CONVEXITy OF THE ChOQUET INTEGRaL}

Let $S=\left\{s_{1}, \ldots, s_{n}\right\}$ be a finite nonempty set of states (of nature). Finiteness of $S$ is assumed for simplicity of exposition; the appendix will indicate that the results of this paper also hold for infinite $S . \Gamma$ is an arbitrary fixed nondegenerate interval. $\mathscr{G}$ is the set of functions from $S$ to $\Gamma$. Some results in the sequel will be formulated for $\mathscr{G}$, some for $\mathfrak{R}^{s}$.

A function $v: 2^{S} \rightarrow[0,1]$ is a capacity if $v(\varnothing)=0, v(S)=1$, and $v$ is monotonic w.r.t. set-inclusion, i.e., $A \supset B \Rightarrow v(A) \geqslant v(B)$. A capacity is superadditive if for all $A, B \subset S, v(A \cup B)+v(A \cap B) \geqslant v(A)+v(B)$. It is subadditive if above we have $\leqslant$ instead of $\geqslant$.

For a capacity $v$, and a function $\varphi: S \rightarrow \mathfrak{R}$, the Choquet integral of $\varphi$ (with respect to $v$ ), denoted $\int_{S} \varphi d v$, or $\int \varphi d v$, is

$$
\int_{0}^{\infty} v(\{s \in S: \varphi(s) \geqslant \tau\}) d \tau+\int_{-\infty}^{0}[v(\{s \in S: \varphi(s) \geqslant \tau\})-1] d \tau .
$$

In [18, Chap. VI] elucidations and illustrations are given. Obviously $\mathscr{G}$ can be considered a convex subset of $\Re^{n}$, and the Choquet integral is a function ("functional") from $\mathfrak{R}^{n}$ or $\mathscr{G}$ to $\mathfrak{R}$. So convexity and concavity of the Choquet integral can be defined as usual. It is straightforward that the Choquet integral is convex/concave on $\mathfrak{R}^{n}$ if and only if it is so on $\mathscr{G}$, so in the sequel we shall freely interchange these. For $\Gamma=\Re$ the following lemma is well-known, see for instance $[5,54.2]$, [10, Propositions 10.2.5 and 10.2.1], [15, the Proposition], or [1, pp. 248/249]. Adaptation to general nondegenerate intervals $\Gamma$ is straightforward (e.g., first for the case where 0 is in the interior of $\Gamma$, using positive homogeneity), and is left to the reader.

Lemma 1. The Choquet integral is concave if and only if the capacity is superadditive, and convex if and only if the capacity is subadditive. This also applies to the restriction of the Choquet integral to $\mathscr{G}$. 
Two functions $\varphi, \psi$ from $S$ to $\Re$ are comonotonic if there do not exist states $s_{i}, s_{j}$ such that

$$
\varphi\left(s_{i}\right)>\varphi\left(s_{j}\right) \quad \text { and } \quad \psi\left(s_{i}\right)<\psi\left(s_{j}\right) .
$$

A set of functions from $S$ to $\Re$ is comonotonic if every pair contained in it is comonotonic. For comonotonic functions $\varphi, \psi$ we have

$$
\int(\alpha \varphi+(1-\alpha) \psi) d v=\alpha \int \varphi d v+(1-\alpha) \int \psi d v
$$

This was proved in [6] (for continuous capacities and maximal algebras) and in [21] (in full generality). The following characterization of concave/convex Choquet integrals will be used in the sequel.

Lemma 2. The Choquet integral is concave if and only if, for all $\varphi, \psi, \xi \in \mathscr{G}$ with $\psi, \xi$ comonotonic and $0<\alpha<1$

$$
\int \varphi d v>\int \psi d v \Rightarrow \int(\alpha \varphi+(1-\alpha) \xi) d v>\int(\alpha \psi+(1-\alpha) \xi) d v .
$$

Above we may replace "concave" by "convex" if we replace " $\psi$, $\xi$ comonotonic" by " $\varphi, \xi$ comonotonic".

Proof. The case of convex Choquet integral is analogous to the case of concave Choquet integral, so we consider only the latter. The only-if part is direct from concavity, positiveness of $\alpha$, and (1). Next we suppose (2) holds, and derive concavity. Let, with "int" denoting topological interior, $\varphi: S \rightarrow \operatorname{int}(\Gamma)$ be an element from $\mathscr{G}$. Identifying $\psi \in \operatorname{int}(\Gamma)$ with a constant function, noting that a constant function is comonotonic with every other function, and applying (2) and (1), we get, with $0<\alpha<1, \xi \in \mathscr{G}$,

$$
\begin{aligned}
\int \varphi d v & >\psi \Rightarrow \int(\alpha \varphi+(1-\alpha) \xi) d v>\int(\alpha \psi+(1-\alpha) \xi) d v \\
& =\alpha \psi+(1-\alpha) \int \xi d v .
\end{aligned}
$$

Taking the supremum over $\psi$ while noting that, by finiteness of $S$, $\int \varphi d v \in \operatorname{int}(\Gamma)$ so that $\int \varphi d v=\sup \left\{\psi \in \operatorname{int}(\Gamma): \psi<\int \varphi d v\right\}$, (3) gives $\int(\alpha \varphi+(1-\alpha) \xi) d v \geqslant \alpha \int \varphi d v+(1-\alpha) \int \xi d v$. I.e., the Choquet integral is concave for acts with image within int $(\Gamma)$. It must be concave. 


\section{SCHMEIDLER's Characterization of ChOQuet Expected Utiltty}

Let $\mathscr{P}$ be the set of money lotteries. I.e., an element $P=$ $\left(p_{1} ; x_{1}, \ldots, p_{m} ; x_{m}\right)$ from $\mathscr{P}$ is a probability distribution with finite support over $\Re$, resulting with probability $p_{1}{ }^{1}$ in the amount of money (real number) $x_{1}, \ldots$, and with probability $p_{m}$ in the amount of money (real number) $x_{m}$. Of course, to any $A \subset \mathfrak{R}, P$ assigns probability $\sum_{x_{i} \in A} p_{i}$. Money lotteries can be "mixed", for $0 \leqslant \alpha \leqslant 1, \alpha P^{1}+(1-\alpha) P^{2}$ assigns $\alpha P^{1}(A)+$ $(1-\alpha) P^{2}(A)$ to $A \subset \mathfrak{R}$.

By $\mathscr{F}$ we denote the set of functions from $S$ to $\mathscr{P}$. Elements of $\mathscr{F}$ are acts. Acts can also be mixed, in a "pointwise manner", as $\alpha f+(1-\alpha) g$ : $s \mapsto \alpha f(s)+(1-\alpha) g(s)$. Finally, $\geqslant$ denotes the preference relation of a decision maker on $\mathscr{F}$.

As usual, we write $f \succ g$ if $f \geqslant g$ and not $g \geqslant f, f \preccurlyeq g$ if $g \geqslant f, f \prec g$ if $g \succ f$, and $f \simeq g$ if $f \geqslant g$ and $g \geqslant f$. Further $\geqslant$ is a weak order if it is complete $(f \geqslant g$ or $g \geqslant f$ for all $f, g)$ and transitive, and $\geqslant$ is trivial if $f \geqslant g$ for all $f, g$. We identify money lotteries with constant acts, and write $P^{1} \geqslant P^{2}$ if $f \succcurlyeq g$ for the act $f$ constant $P^{1}$ and $g$ constant $P^{2}$; analogously for $\succ, \preccurlyeq$, $\prec, \simeq$. This induces preference relations over money lotteries. A function $V: \mathscr{F} \rightarrow \mathfrak{R}$ represents $\geqslant$ if, for all acts $f, \dot{g}$,

$$
f \geqslant g \Leftrightarrow V(f) \geqslant V(g) .
$$

We say Choquet expected utility applies if there exist a capacity $v$ on $S$, and a utility function $u: \mathscr{P} \rightarrow \Re$, such that $f \mapsto \int u \circ f d v$ (the Choquet integral of $u \circ f$ ) represents $\geqslant$.

We assume that the probability mechanism which generates money lotteries gives well-established probabilities, not inducing any optimism or pessimism. Hence we shall, like Anscombe and Aumann [2] and Schmeidler [15], assume in the sequel that the decision maker uses expected utility to value these lotteries. Then $u$, the utility function over the money lotteries, will be an expected utility function, i.e., it is linear. $(\mathscr{P}$ can be considered a convex subset of a linear space so this is well-defined.) We can then define $U: \mathfrak{R} \rightarrow \mathfrak{R}$ by $U: \mu \mapsto u(1 ; \mu)$, and get $u\left(p_{1} ; x_{1}, \ldots, p_{m} ; x_{m}\right)$ $=\sum_{i=1}^{m} p_{i} U\left(x_{i}\right)$. Because of this we shall then write $E U$ instead of $u$. Our central interest will be the way in which the decision maker processes the, unprobabilized, uncertainty concerning the true state. Here we will incorporate the phenomenon of optimism/pessimism, reflected through nonadditivity of the capacity. $[7 ; 17 ; 18$, Chap. VI; 12] derive Choquet expected utility without probability mechanisms.

First we adapt the definition of comonotonicity, given before for func-

${ }^{1}$ Or, more accurately, $\sum_{x_{i}=x_{1}} p_{i}$. 
tions from $S$ to $\mathfrak{R}$, to acts. It can be seen that under Choquet-expected utility two acts $f, g$ are comonotonic if and only if the functions $u \circ f, u \circ g$ are comonotonic.

Definmon 3 (see Fig. 1 below). Acts $f$ and $g$ are comonotonic if there do not exist states $s_{i}$ and $s_{j}$ so that $f\left(s_{i}\right)>f\left(s_{j}\right)$ and $g\left(s_{i}\right) \prec g\left(s_{j}\right)$.

A set of acts is comonotonic if every pair of acts in the set is comonotonic. Obviously every constant act is comonotonic with any other act. The following definition gives the well-known independence condition for (additive, von Neumann-Morgenstern) utility, the earliest reference for which is Marschak [11] so far as we know (see also the loose-hand [14]). Further the definition gives comonotonic independence as introduced in $[15]$.

Definition 4 (See Fig. 2 below). We say $\succcurlyeq$ satisfies (mixture-) independence if, for all acts $\{f, g, h\}$, and $0<\alpha<1$,

$$
f \succ g \Rightarrow \alpha f+(1-\alpha) h \succ \alpha g+(1-\alpha) h .
$$

We say $\geqslant$ satisfies comonotonic independence if implication (4) is required only when $\{f, g, h\}$ is comonotonic.

Elucidation of comonotonic independence will be given in Section 4. [15] proved the following result (also for infinite state spaces, see the Appendix ). [19, Sect. 5] gave an alternative self-contained proof, not using results from functional analysis ("fuzzy measure" is mathematically identical to capacity). We give two preparatory definitions. In the first "vNM" abbreviates "von Neumann-Morgenstern".

We call $\geqslant(v N M-)$ continuous if, for all acts $f \succ g>h$, there exist $\alpha$ such that $(1-\alpha) f+\alpha h \succ g$, and $\beta$ such that $g \succ \beta f+(1-\beta) h$.

We call $\geqslant$ weakly monotonic if, for all acts $f, g$,

$$
f(s) \geqslant g(s) \text { for all } s \Rightarrow f \geqslant g .
$$

THEOREM 5 (Schmeidler). Let $\mathscr{P}$ be the set of money lotteries, $S$ a finite set of states, $\mathscr{F}$ the set of functions from $S$ to $\mathscr{P}$, and $\geqslant$ a nontrivial preference relation on $\mathscr{F}$. The following two statements are equivalent:

(i) There exist a capacity $v$ on $S$, and a function $U: \Re \rightarrow \mathfrak{R}$, such that $f \mapsto \int_{S} E U \circ f d v$ represents $\succcurlyeq$.

(ii) The preference relation $\geqslant$ is a weakly monotonic VNM-continuous weak order, satisfying comonotonic independence.

Further, $v$ in (i) is uniquely determined, and $U$ is cardinal. ${ }^{2}$

${ }^{2}$ This abbreviates: "unique up to a positive linear transformation." 
The theorem of Anscombe and Aumann [2] results if in (i) above the requirement is added that $v$ is additive, and in (ii) above comonotonic independence is strengthened to mixture-independence. In the context where lotteries are for money it is natural to add nondecreasingness of $U$. This can be characterized by adding in (ii) above monotonicity of $\geqslant$ on $\mathscr{P}$ w.r.t. stochastic dominance. Continuity of $U$ is central in [17], [18, Chapter VI].

\section{Pessimism- ANd OPTIMISM-INDEPENDENCE}

To suggest intuitively how pessimism can be characterized directly through comonotonicity, we consider the phenomenon of "hedging" as described in Yaari [23] ("bets on the same event") and Yaari [24, p. 104]. (See Fig. 1.)

Suppose two acts $f, g$ are not comonotonic, so that there exist two states, say $s_{1}, s_{2}$, with $f\left(s_{1}\right) \succ f\left(s_{2}\right)$ and $g\left(s_{1}\right) \prec g\left(s_{2}\right)$. Say $E U\left(f\left(s_{1}\right)\right)=17$, $E U\left(f\left(s_{2}\right)\right)=13, E U\left(g\left(s_{1}\right)\right)=1, E U\left(g\left(s_{2}\right)\right)=7$. See Fig. 1b. The mixture $\frac{1}{2} f+\frac{1}{2} g$ assigns $E U$-value 9 to $s_{1}$ and 10 to $s_{2}$. It seems that the mixing has induced a reduction of the involved uncertainty. At $s_{1}, f\left(s_{1}\right)$ was relatively favorable (favorable as compared to $f\left(s_{2}\right)$ ), this provided a hedge against the rclativcly unfavorable $g\left(s_{1}\right)$ (unfavorable as compared to $g\left(s_{2}\right)$ ). A same hedge, with $f$ and $g$ interchanged, occurred at $s_{2}$.

The hedging described above is typically a phenomenon that will occur
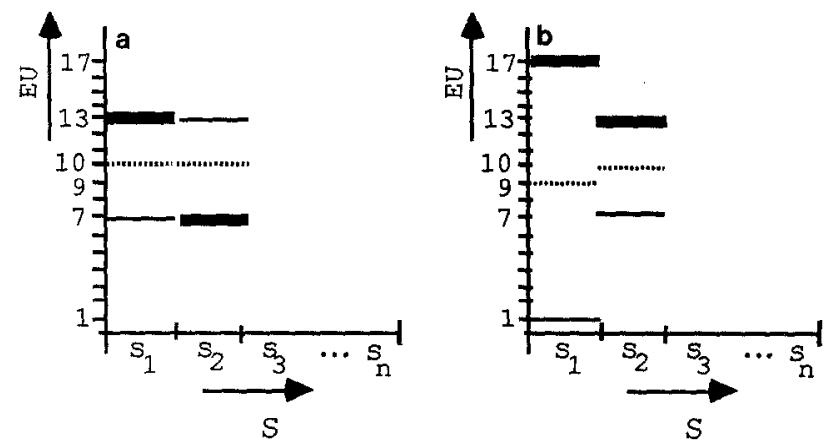

FIG. 1. Comonotonicity and hedging. - $: E U(f) ;-: E U(g) ; \cdots: E U\left(\frac{1}{2} f+\frac{1}{2} g\right)$. Both in Fig. $1 \mathrm{a}$ and in Fig. $1 \mathrm{~b}, f$ and $g$ are not comonotonic since for $f$ state $s_{1}$ is strictly more favorable than $s_{2}$, whereas for $g$ this is reversed, $s_{2}$ is strictly more favorable than $s_{1}$. In the mixture $\frac{1}{2} f+\frac{1}{2} g$ the (relatively) favorable outcome of $f$ at $s_{1}$ gives a hedge against the relatively unfavorable outcome of $g$ at $s_{1}$; at $s_{2}$ the same things apply with $f$ and $g$ interchanged. The mixture $\frac{1}{2} f+\frac{1}{2} g$ seems to involve less uncertainty than $f$, and also less than $g$. 
if and only if two acts are not comonotonic. It is a kind of reduction of the involved uncertainty. Comonotonic independence wants to allow for hedging effects, hence the implication (4) of mixture-independence is required only when, through comonotonicity, it has been guaranteed that no "disturbing" hedging effects will occur.

This being understood, the intuition underlying the characterization of pessimism (and, analogously, optimism) comes to mind easily. A pessimist dislikes uncertainty, hence the reduction of uncertainty through hedging will lead to additional appreciation. An optimist, who expects uncertainty to turn out favorably, will not appreciate the reduction of uncertainty through hedging. So we formulate:

Defintion 6 (See Fig. 2). $\succcurlyeq$ satisfies pessimism-independence if implication (4) is required only when $\{g, h\}$ is comonotonic, and $\geqslant$ satisfies optimism-independence if implication (4) is required only when $\{f, h\}$ is comonotonic.

Indeed, for a pessimist noncomonotonicity of $\{f, h\}$ will only induce an additional appreciation of the mixture $\alpha f+(1-\alpha) h$, so a strengthening of
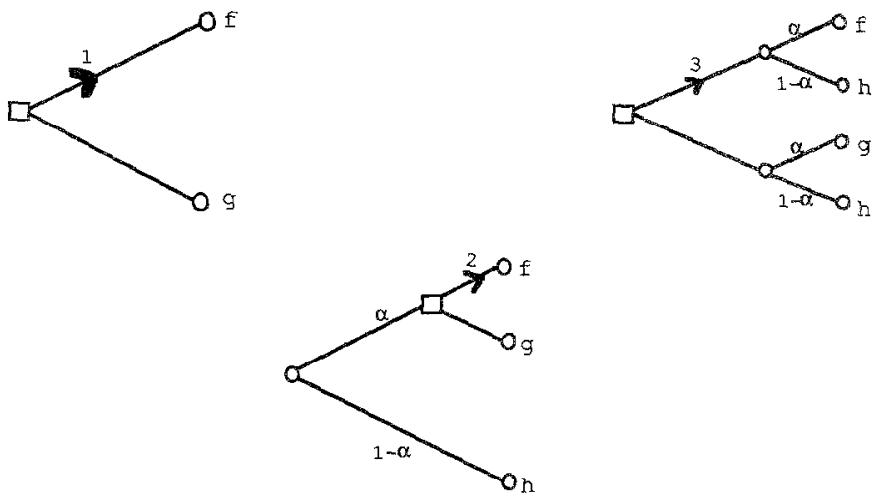

FiG. 2. Independence. In $\square$-points the decision maker must choose; in $C$-points chance (say a roulette wheel) chooses. The arrows $>$ indicate preferences. According to mixtureindependence the (very bold) preference 1 should imply preference 3. Comonotonic independence requires the implication of preference 3 by preference 1 only if all involved acts are comonotonic. The idea is that, because of vagueness, the mixing of acts may induce "disturbing" hedging effects. Hence, the implication is required only if hedging effects are excluded by comonotonicity. According to pessimism-independence hedging is always appreciated, so for noncomonotonic $f, h$ the above implication should also hold as long as $g, h$ are comonotonic. According to optimism-independence hedging is never appreciated; hence only acts $f$ and $h$ need to be comonotonic. 
the preference. For an optimist noncomonotonicity of $\{g, h\}$ will only induce an additional depreciation of the mixture $\alpha g+(1-\alpha) h$, so again a strengthening of the preference.

For understanding of the difference between the above definition and the previous Definition 4 it is good to note that comonotonicity of $\{f, g\}$ is relevant in none of these definitions. It may be added or deleted in each of the definitions without affecting the implications. [19, Observation 20] indicated, even stronger, that the implication (4) is needed only for the case where either $f$ or $g$ is constant.

The following lemma contains the main new result of this paper.

Lemma 7. Let Choquet expected utility apply, with $u$ linear and nonconstant. Then the capacity $v$ is superadditive if and only if pessimismindependence applies, and it is subadditive if and only if optimismindependence applies.

Proof. Set $\Gamma:=u(\mathscr{P}) . \Gamma$ is nondegenerate and convex. For every function $\varphi: S \rightarrow \Gamma$ there exists an act $f$ such that $E U \circ f=\varphi$. Comonotonicity of acts $f, g$ corresponds with comonotonicity of the functions $E U \circ f, E U \circ g$. The lemma now follows from Lemmas 1 and 2.

COROLlaRY 8. In Theorem 5 we may add in statement (i) that $v$ is superadditive if in (ii) we strengthen comonotonic independence to pessimismindependence. We may add in statement (i) that $v$ is subadditive if in (ii) we strengthen comonotonic independence to optimism-independence.

Yaari [24] characterized a nonlinear-probability approach to decision making under risk, where an objective probability measure $P$ is given in advance. $P$ is then transformed into a capacity, with the transformation a nondecreasing and continuous function from the unit interval onto the unit interval. Completely analogously to the above corollary, in Yaari's approach optimism/pessimism can be characterized directly in terms of comonotonicity. We do not elaborate all details; the main issues are discussed in the Appendix.

COROLLARY 9. In Theorem 1 in Yaari [24] we may add convexity of the transformation (" $f$ " in Yaari's notation) if we replace Axiom A5 by the (according to Yaari's Proposition 3 equivalent, and economically more meaningful ) Axiom A5*, and require comonotonicity only for $v, w$ there; we may add concavity of the transformation if we replace Axiom A5 by Axiom A5*, and require comonotonicity only for $u, w$ there. 
TABLE I

\begin{tabular}{l|l|l|} 
& $f, h$ comonotonic & $f, h$ generai \\
\hline$g, h$ comonotonic & $v$ general capacity & $v$ superadditive \\
\hline$g, h$ general & $v$ subadditive & $v$ additive (prob. meas.) \\
\hline
\end{tabular}

Note. Let the preference relation be a weak order and satisfy vNM-continuity. Then for each entry in the matrix the following holds: Choyuet expected utility applies, with the capacity as described in the entry, if and only if the "independence" implication $[0<\alpha<1$, $f \succ g \Rightarrow \alpha f+(1-\alpha) h \succ \alpha g+(1-\alpha) h]$ holds for those $f, g, h$ that satisfy the restrictions applying to the involved array and column. (Imposing comonotonicity of $f, g$ never adds a real restriction; see text above Lemma 7 .)

The results are summarized in Table $\mathrm{I}$.

\section{APPENDIX}

[19, Theorem 11] strengthened Theorem 5 by weakening comonotonic independence to "maxmin-independence" in statement (ii). Corollary 8 also holds for that case, with an identical proof.

[15] extended Theorem 5 to infinite state spaces, for "strongly bounded" acts; [22] gave the extension to unbounded acts, and mentioned and solved some problems about measurability in comonotonic independence. For these works the results of this note remain valid. [24] requires infiniteness of the state space. Again, this does not affect our resuits.

The only structure of $\mathscr{P}$ we used above was that it was a mixture space as defined in [9], w.r.t. which $E U$ was linear. (It was for instance not at all essential that the lotteries were for money.) Hence we can directly apply the above analysis to the case of [24], with acts assigning amounts of money instead of money lotteries to states and with again utility linear. Yaari considers the case where an objective probability $P$ is transformed in a nondecreasing way ("anticipated utility"). As Yaari indicated on p. 114, this means that he is considering Choquet-expected utility with as capacity the transformed probability measure, $f \circ P$ in Yaari's notation. ([20] shows that Choquet expected utility, when adopted in decision making under risk, in fact is identical to (Quiggin/)Yaari's anticipated utility.) Our results can be applied to Yaari's approach, characterizing for instance superadditivity (the case of subadditivity is analogous and will not be elaborated) of the transformed probability measure. It remains to be shown that that is equivalent to convexity of the transformation. It is straightforward to show that convexity of the transformation implies superadditivity of the transformed probability measure. The reversed implication, in general, does not have to hold. It may be impossible to have $\varphi$ convex. It does however 
hold in the set-up of Yaari, as the following lemma shows. Note that the existence of atoms is excluded by Yaari's "richness" requirement that all probability distributions can be generated.

LEMMA 10. Let $(S, \mathscr{A}, P)$ be an atomless probability space (with $\mathscr{A}$ a $\sigma$-algebra, and $P$ o-additive). Let, for some nondecreasing $\varphi:[0,1] \rightarrow$ $[0,1], \varphi \circ P$ be superadditive. Then $\varphi$ is convex.

Proof. By [8, Theorems 86, 111, where "convexity" means midpoint convexity] it is sufficient for convexity of the nondecreasing $\varphi$ that $\varphi$ satisfy midpoint convexity. I.e., for $0 \leqslant \alpha \leqslant \beta \leqslant 1$ we must prove that $\varphi\left(\frac{1}{2} \alpha+\frac{1}{2} \beta\right) \leqslant \frac{1}{2} \varphi(\alpha)+\frac{1}{2} \varphi(\beta)$. By nonatomicity we can find (see for instance [16]) "nested" events $A \subset B \subset C$ with $P(A)=\alpha, P(B)=\frac{1}{2} \alpha+\frac{1}{2} \beta, P(C)=\beta$. Supperadditivity gives $\varphi(P(C))+\varphi(P(A)) \geqslant \varphi(P(B))+\varphi(P((C-B) \cup A))$. Noting that $P(B)=P((C-B) \cup A)$, one gets midpoint convexity.

If we combine the maxmin idea mentioned in the first paragraph of the Appendix and the real-number linear utility set-up of [24], then the resulting version of pessimism-independence can be seen to be related to the uncertainty-aversion axioms A.5 and A.6 in [3], axioms which have inspired us.

\section{ACKNOWLEDGMENT}

The presentation of the paper was improved by the comments of an anonymous referee.

\section{REFERENCES}

1. B. ANGER, Representations of capacities, Math. Ann. 229 (1977), 245-258.

2. F. J. Anscombe and R. J. AumanN, A definition of subjective probability, Ann. Math. Statist. 34 (1963), 199-205.

3. A. Chateauneuf, Uncertainty aversion and risk aversion in models with nonadditive probabilities, in "Risk, Decision and Rationality," (B. R. Munier, Ed.), pp. 615-629, Reidel, Dordrecht, 1988.

4. S. H. CHEW, E. KARN, AND Z. SAFRA, Risk aversion in the theory of expected utility with rank dependent probabilities, J. Econ. Theory 42 (1987), 370-381.

5. G. Chopuet, Theory of capacities, Ann. Inst. Fourier (Grenoble) 5 (1953-1954), 131-295.

6. C. Dellacherie, Quelques commentaires sur les prolongements de capacites, in "Seminaire de Probabilités V Strasbourg," Lecture Notes in Mathematics, Vol. 191, SpringerVerlag, Berlin/New York, 1970.

7. I. Gilbos, Expected utility with purely subjective non-additive probabilities, J. Math. Econ. 16 (1987), 65-88.

8. G. H. Hardy, J. E. Littlewood, and G. Pólya, "Inequalities," Cambridge Univ. Press, Cambridge, 1934; second ed. 1952, reprinted 1978.

9. 1. N. HERSTEIN AND J. MILNOR, An axiomatic approach to measurable utility, Econometrica 21 (1953), 291-297. 
10. P. J. Huber, "Robust Statistics," Wiley, New York, 1981.

11. J. MARSCHAK, Rational behavior, uncertain prospects, and measurable utility, Econometrica 18 (1950), 111-141.

12. Y. Nakamura, Subjective expected utility with non-additive probabilities on finite state space, Discussion Paper No. 400, University of Tsukuba, Tsukuba, Ibaraki, Japan, 1989.

13. J. Quigarn, A theory of anticipated utility, J. Econ. Behav. Organ. 3 (1982), 323-343.

14. H. Rubin, Postulates for the existence of measurable utility and psychological probability (Abstract 493), Bull. Amer. Math. Soc. 55 (1949), 1050-1051.

15. D. SCHMEIDLER, Subjective probability and expected utility withou additivity, Econometrica 57 (1989), 571-587.

16. C. VILlegAS, On quantitative probability $\sigma$-algebras, Ann. Math. Statist. 35 (1964), $1787-1796$.

17. P. P. WAKKER, Continuous subjective expected utility with nonadditive probabilities, J. Math. Econ. 18 (1989), 1-27.

18. P. P. WAKKER, "Additive Representations of Preferences, A New Foundation of Decision Analysis," Kluwer, Dordrecht, 1989.

19. P. P. WAKker, A behavioral foundation for fuzzy measures, Fuzzy Sets and Systems, forthcoming.

20. P. P. WAKKER, Under stochastic dominance Choquet-expected utility and anticipated utility are identical, Theory and Decision, forthcoming.

21. P. P. WAKKER, "Comonotonic Additivity for Choquet Integrals," Internal Report 89 NICI 11, University of Nijmegen, NICI, Department of Mathematical Psychology, Nijmegen, The Netherlands, 1989.

22. P. P. WAKKER, "From Finite- to Infinite-Dimensional Integral Representations; Unbounded Utility for Savage (1954) and Others," Fuqua School of Business, Duke University, Working Paper 8928, 1989.

23. M. E. YAARI, Some remarks on measures of risk aversion and on their uses, $J$, Econ Theory 1 (1969), 315-329.

24. M. E. YAARI, The dual theory of choice under risk, Econometrica $55(1987), 95-115$. 